\title{
The Posterior Cerebral Artery and its Main Cortical Branches Identified with Noninvasive Transcranial Color-Coded Duplex Sonography
}

Authors

Affiliations
P. E. Frid ${ }^{\text {}, ~ S . ~ J . ~ S c h r e i b e r ~}{ }^{2}$, O. Pade ${ }^{3}$, F. Doepp ${ }^{4}$, J. Valdueza

Affiliation addresses are listed at the end of the article
Key words

- brain

- blood vessels

ultrasound received 24.06.2015

accepted $\quad 12.10 .2015$

Bibliography

DOI http://dx.doi.org/

10.1055/s-0035-1565130

Published online:

November 6, 2015

Ultrasound International Open

2015; 1: E53-E57

(c) Georg Thieme Verlag KG

Stuttgart · New York

ISSN 2199-7152

Correspondence

Dr. Petrea E Frid, MD

Department of Neurology Institute for Clinical Sciences

Skåne University Hospital

Malmö

Sweden S-20502

Tel.: + 46/731/500943

Fax: + 46/403/33 055

petrea.frid@med.lu.se

\section{Abstract}

$\nabla$

Purpose: To differentiate PCA segments and cortical branches by means of transcranial colorcoded duplex sonography (TCCD) and to measure flow parameters at rest and during visual stimulation.

Materials and Methods: 60 healthy subjects with a good acoustic temporal bone window were examined. The main stem of the PCA (P1, P2 and $\mathrm{P} 3$ ) and 4 main cortical branches - the anterior temporal artery (ATA), the occipital temporal artery (OTA), the parietooccipital artery (POA) and the calcarine artery (CA) - were assessed using an axial transtemporal approach. Systolic and diastolic blood flow velocities (BFVs) were recorded at rest and during visual stimulation.

Results: Identification of the P1 segment of the PCA was successful in $97.5 \%(117 / 120)$ of cases.

\section{Introduction}

Infarctions in the territory of the posterior cerebral artery (PCA) are reported in $5-10 \%$ of ischemic strokes [1]. Main causes are thought to be embolic occlusions, but intrinsic vessel stenosis and occlusions may occur [2]. Despite the clinical relevance, less attention has been paid to the specific vessel anatomy of the PCA. For clinical use the PCA can be divided into 4 segments from P1 to P4 [3]. P1 represents its shortest and most proximal segment, with a mean length of less than $10 \mathrm{~mm}$, [4-6] running from its origin at the top of the BA to its side-to-end anastomosis with the posterior communicating artery (PCoA). $\mathrm{P} 2$ begins at the junction with PCoA and has the longest course. It can be further subdivided into an anterior (proximal) and posterior (distal) part, each about $25 \mathrm{~mm}$ in length [5]. P3 begins at the posterior margin of the midbrain and runs slightly upward and medially within the quadri-
The P2 and P3 segments were visualized in all cases. The 4 main cortical branches could be identified to varying degrees: ATA in $88 \%$, OTA in $96 \%, \mathrm{POA}$ in $69 \%$ and $\mathrm{CA}$ in $62 \%$. There was an evoked flow response in the P2 main stem and in all cortical branches. The most pronounced increase in diastolic/systolic BFV after visual stimulation test was seen in the CA $(42 \% / 35 \%)$, followed by P2 (30\%/24\%), the POA $(27 \% / 27 \%)$, the OTA (16\%/13\%) and the ATA (9\%/8\%).

Conclusion: Insonation through the temporal bone window with TCCD confidently allows the assessment of the P1 to P3 segments of the PCA as well as the 2 proximal branches, the ATA and the OTA. An ultrasound-based classification of PCA anatomy and its cortical branches may be used as a noninvasive method for the evaluation of posterior circulation pathology.

geminal cistern. It reaches the medial surface of the occipital lobe, often ending at the anterior limit of the calcarine fissure. At this point $\mathrm{P} 3 \mathrm{usu}$ ally divides into 2 major branches defining P4; the calcarine artery (CA) and the parietooccipital artery (POA). This distal bifurcation can be found at varying depths ranging from 6 to $67 \mathrm{~mm}$ ( $\bullet$ Fig. 1-3).

The branching pattern of the cortical arteries of the PCA has been mapped by several groups. The number of proper arterial branches mentioned in the literature varies depending on the method of investigation and the study material $[5,7]$. Zeal and Rhoton anatomically identified 7 main arterial branches that occur with varying consistency [5]. The most constantly developed arteries are the anterior temporal artery (ATA) arising from the anterior $(64 \%)$ or posterior $(20 \%)$ part of $P 2$, and the occipitotemporal artery (OTA) (synonym: posterior temporal artery) most frequently arising from the distal segment of P2. The OTA is a 

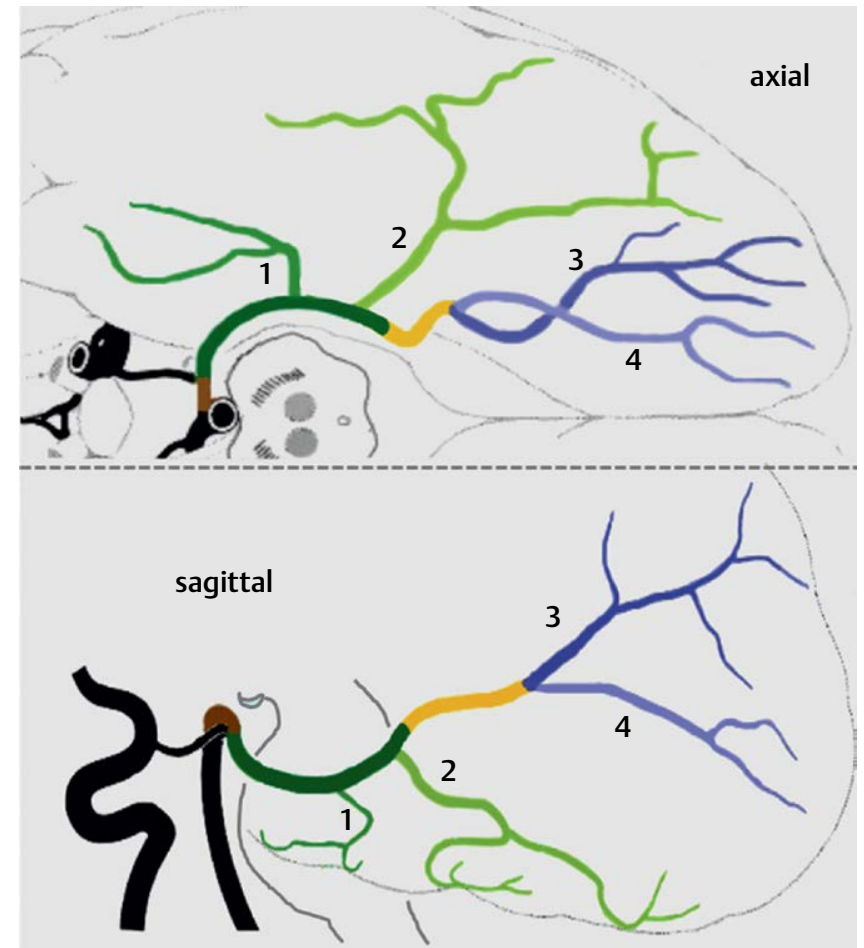

Fig. 1 Schematic drawing of PCA segments (adapted from Huber) in the axial view (top) and the sagittal view (bottom): Brown $=\mathrm{P} 1$ segment, dark green $=\mathrm{P} 2$ segment, yellow $=\mathrm{P} 3$ segment, $1=$ anterior temporal artery, 2 =occipitotemporal artery, 3 = parietooccipital artery, 4 = calcarine artery.

firm branch coursing in a posterolateral direction, supplying the inferior temporal and occipital surfaces and the lingual gyrus. The POA occurs as a single terminal branch, in about half of the cases arising from $\mathrm{P} 3$ as part of a terminal bifurcation with the CA. In $40 \%$ of cases it branches off the distal P2 and in $10 \%$ even from the proximal P2 [5]. From its medial origin the POA rises upward and laterally to the parietooccipital fissure and during its course often crosses the proximal calcarine fissure. The CA is the second terminal PCA branch. In about half of cases it arises from P3 but may depart from the distal P2 in $42 \%$ of cases. In $10 \%$ it may also branch off the POA [5]. Its irrigation area includes, but is not limited to, the visual cortex.

MRA and CTA techniques also allow good visualization of the major PCA branches, but no detailed assessment has been reported up to now $[8,9]$, and to our knowledge, no previous studies on flow rates measured with TCCD have been previously published. Early sonographic PCA assessments using transcranial Doppler (TCD) divided the PCA into a P1 and P2 segment, based on the detected flow direction. A flow direction towards the transducer was defined as the P1 segment, and a flow away from the transducer as the P2 segment [10]. However, this nomenclature does not correspond to real anatomy. TCCD allows a more precise anatomical correlation and PCA assessment in relation to its junction with the PCOA. Using TCCD, the proximal P2 shows - identical to P1 - a flow direction towards the transducer and the distal P2 and P3 and P4 show a flow away from the transducer [11]. Based on anatomical data, we analyzed the main PCA segments as well as the PCA branches ATA, OTA, POA and $C A$, the latter of which have not yet been described and classified with TCCD. In addition, blood flow in the P2 segment as well as in the 4 cortical branches was analyzed during a visual stimulation test to prove the hypothesis that the magnitude of
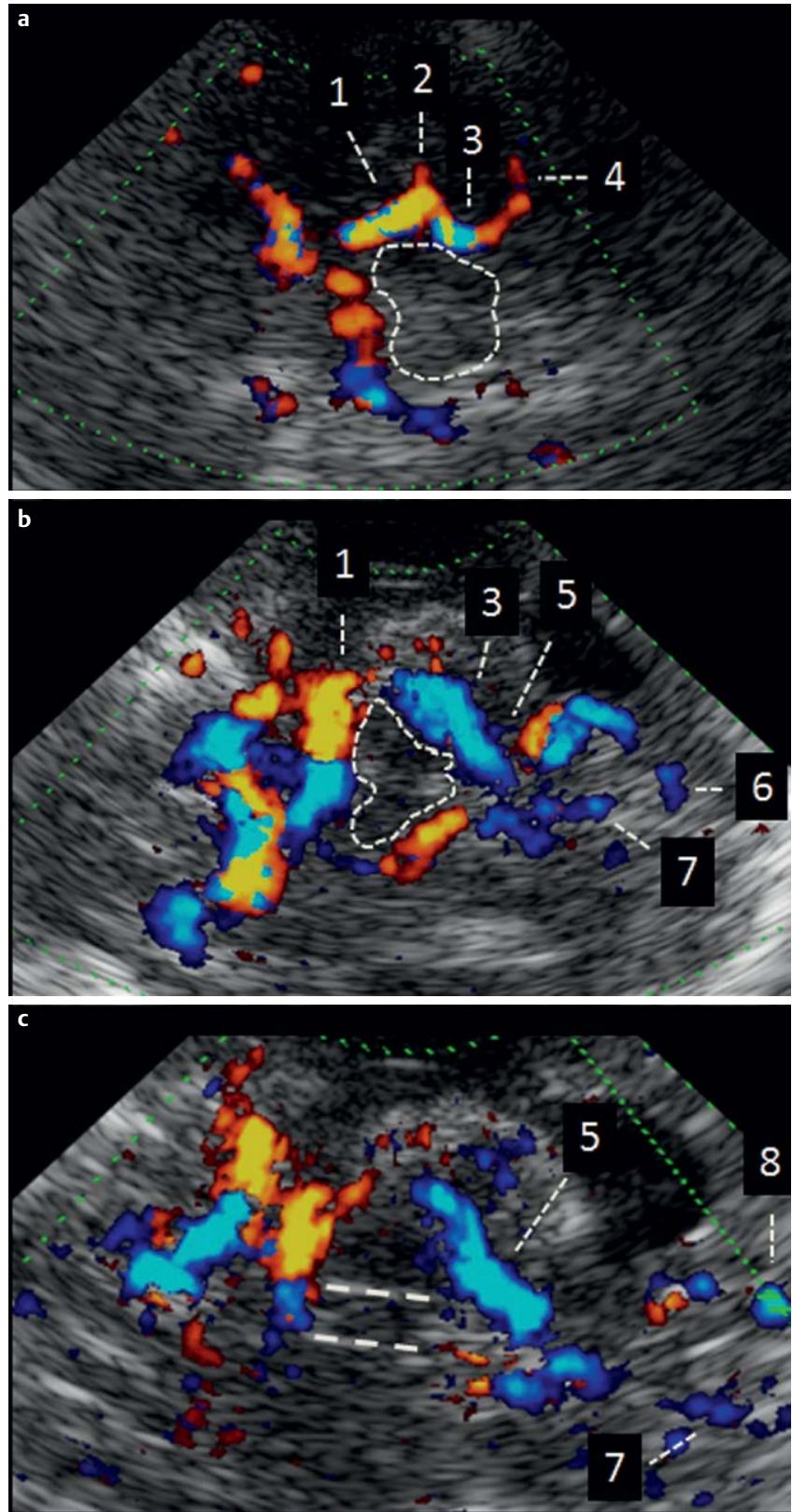

Fig. 2 TCCD, color mode images, axial planes. a pontomesencephalic plane, $\mathbf{b}$ mesencephalic plane, $\mathbf{c}$ thalamic plane. Red indicates flow toward the probe, blue indicates flow away from the probe. Midbrain encircled in white. The third ventricle is outlined by white dashed lines. $1=$ proximal P2 segment, 2 = anterior temporal artery, 3 = distal P2 segment, 4 = occipitotemporal artery, 5=P3 segment, 6 = calcarine artery, 7 = straight sinus, $8=$ parietooccipital artery.

responses relate to each vessel's participation in feeding the striatal and peristriatal visual cortex.

\section{Materials and Methods}

$\nabla$

Healthy subjects with a sufficient acoustic temporal bone window, i.e., the M1 segment of the middle cerebral artery (MCA) and the P1 and P2 segment of the PCA were easily visualized on both sides, were recruited at the neurological center of the Segeberger Kliniken in Germany. Subjects with an insufficient acoustic temporal bone window were excluded. Informed written consent was obtained from all subjects. The study was approved 
by the local ethics committee. Subjects were investigated in the supine position without a contrast enhancer. TCCD was performed using a Power Vision 6000 SSA-370A ultrasound system (Toshiba, Tokyo, Japan) with a $2.5-\mathrm{MHz}$ phased-array transducer. All vessels were examined using an axial insonation plane through the temporal bone window, which is most easily achieved by placing the probe directly preauricularly and superior to the zygomatic arch. In the mesencephalic plane, the P1 and the proximal P2 were visualized red-colored with a flow towards the transducer. The junction between P1 and P2 was defined by the concomitant visualization of the distal ICA if no PCoA was visible. The origin of the ATA was defined as a redcolored vessel at the junction between the proximal and distal P2 with a flow towards the probe. The origin of the OTA was defined as a comma-shaped, red-colored vessel at the distal P2. Reflecting the slightly upward vessel course, the distal P2 was defined by its blue-colored visualization with a flow away from the probe mainly in a midbrain-thalamic insonation plane. P3 was defined as the main course of the PCA within the quadri-

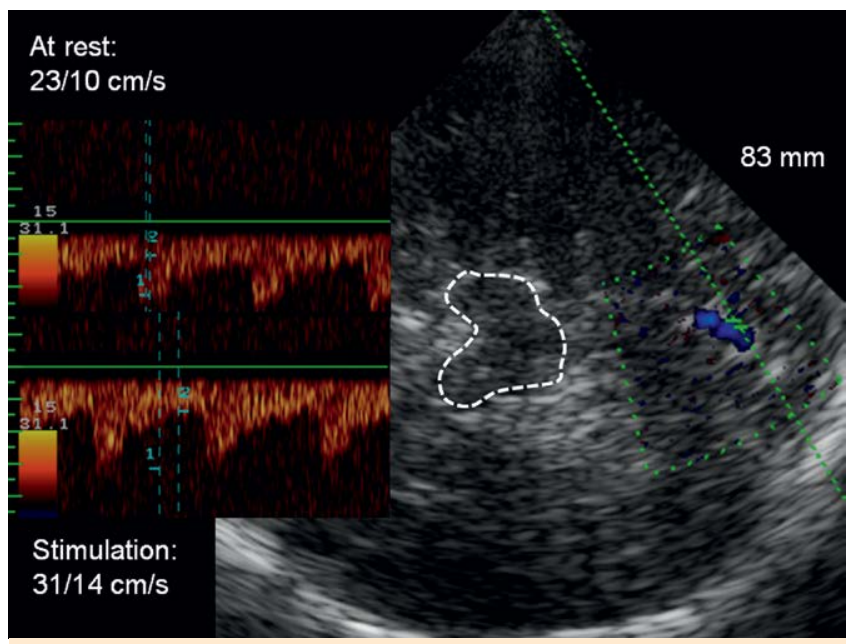

Fig. 3 TCCD, right: color mode image, axial mesencephalic plane, midbrain encircled in white. Calcarine artery detected at a depth of $83 \mathrm{~mm}$. Left: Doppler spectra of the calcarine artery at rest (top) and during visual stimulation (bottom). Note the systolic and diastolic flow velocity increase of $35 \%$ and $40 \%$, respectively.

Table 1 PCA main stem detection rates and flow velocities.

\begin{tabular}{|cccc} 
& Detection rate (\%) & V diast (cm/s) & V syst (cm/s) \\
P1 & 97.5 & $28.1 \pm 8.5(11-52)$ & $59.8 \pm 14.3(27-103)$ \\
P2 & 100 & $27 \pm 6(16-43)$ & $58 \pm 11(39-104)$ \\
P3 & 100 & $29.9 \pm 7.9(18-58)$ & $62.6 \pm 12.0(30-94)$ \\
\hline
\end{tabular}

Mean \pm standard deviation, range ( ), flow velocities without angle correction geminal cistern. A bifurcation in the quadrigeminal cistern or more distal in the thalamic plane was defined as the origin of the POA and CA, both vessels representing P4. A vessel course upward and lateral into the parietooccipital fissure (between the thalamic and the cella media plane) was allocated to the POA. A midbrain-plane vessel course near to the interhemispheric space was allocated to the $C A$. In cases of doubt regarding the differentiation between the POA and CA, the vessel with the stronger response to the visual stimulation test was defined as the $C A$. The systolic and diastolic blood flow velocity (BFV) in the PCA main stem and the cortical branches was measured at rest and during a visual stimulation test. Since the depicted vessels were curved or their length was usually short $(<1 \mathrm{~cm})$, all flow velocity measurements were performed without angle correction. Subjects were examined on both sides. Baseline systolic and diastolic BFVs were measured with the subject's eyes closed. Visual activation was achieved by eye opening and looking at a checker-chart on the ceiling for 20 s. BFVs were measured at the end of the $20 \mathrm{~s}$ and the increase of flow was calculated as the percent increase above baseline levels for all studied vessels. Statistical analysis of the difference in average BFV increase between branches was performed using the Kruskal-Wallis test (non-parametric ANOVA) and multiple comparisons between the 5 groups were performed using Dunn's post-test.

\section{Results}

$\nabla$

60 healthy subjects, aged $18-62$ years (39 \pm 10.9$)$, were included in the study. P1 was successfully identified in $97.5 \%(117 / 120)$ of the cases. P2 and P3 were visualized in all cases. BFVs were almost identical with slightly higher values in P2 and P3 compared with P1 ( $\bullet$ Table 1 ).

Following the defined criteria, the 4 main cortical PCA branches were identified to varying degrees: ATA in $88 \%$ of cases, OTA in $96 \%$ of cases, POA and CA in $69 \%$ and $62 \%$ of cases, respectively. The highest BFVs were measured in the POA, followed by the CA and the OTA with similar values. The ATA showed the lowest BFVs ( $\bullet$ Table 2 ).

Visual stimulation led to a BFV increase in P2 and all 4 cortical PCA branches. The most pronounced increase in diastolic/systolic BFV was found in the CA $(42 \% / 35 \%)$, followed by P2 (30\%/24\%), the POA $(27 \% / 27 \%)$, the OTA (16\%/13\%) and the ATA (9\%/8\%) ( Table 2). Non-parametric ANOVA yielded a significant difference for diastolic and systolic flow increases (KW=123.06 and 106.53, $\mathrm{p}<0.0001)$. Multiple comparisons (systolic and diastolic BFV rise) showed a significant difference between groups $(\mathrm{p}<0.01)$ except for the comparison of PCA vs. POA and ATA vs. OTA.

Table 2 PCA cortical branches: detection rates, flow velocities and response to checker-chart visual stimulation.

\begin{tabular}{|c|c|c|c|c|c|c|c|}
\hline & $\begin{array}{c}\text { Detection rate } \\
\text { (\%) }\end{array}$ & $\begin{array}{l}V \text { diast at rest } \\
(\mathrm{cm} / \mathrm{s})\end{array}$ & $\begin{array}{c}\text { V diast } \\
\text { stimulation }(\mathrm{cm} / \mathrm{s})\end{array}$ & $\begin{array}{c}\text { Change V diast } \\
\text { (\%) }\end{array}$ & $\begin{array}{l}\text { V syst at rest } \\
\qquad(\mathrm{cm} / \mathrm{s})\end{array}$ & $\begin{array}{c}\text { V syst stimulation } \\
\qquad(\mathrm{cm} / \mathrm{s})\end{array}$ & $\begin{array}{c}\text { Change V } \\
\text { syst (\%) }\end{array}$ \\
\hline ATA & 88 & $12 \pm 4(5-2)$ & $13 \pm 5(7-30)$ & 8.5 & $26 \pm 9(12-56)$ & $28 \pm 11(13-63)$ & 8.2 \\
\hline OTA & 96 & $16 \pm 5(8-27)$ & $19 \pm 6(9-35)$ & 16 & $34 \pm 9(18-57)$ & $38 \pm 12(19-70)$ & 12.6 \\
\hline POA & 69 & $21 \pm 8(9-62)$ & $26 \pm 10(10-71)$ & 27.4 & $40 \pm 14(19-100)$ & $51 \pm 18(23-124)$ & 27.1 \\
\hline CA & 62 & $16 \pm 6(5-31)$ & $22 \pm 8(7-47)$ & 41.6 & $33 \pm 10(14-62)$ & $43 \pm 13(19-83)$ & 34.7 \\
\hline P2 & 100 & $27 \pm 6(16-43)$ & $34 \pm 8(20-52)$ & 29.5 & $58 \pm 11(39-104)$ & $72 \pm 13(48-117)$ & 23.9 \\
\hline
\end{tabular}

Mean \pm standard deviation, range ( ), flow velocities without angle correction 


\section{Discussion \\ $\nabla$}

This study shows that the main segments of the PCA as well as the most relevant cortical branches can be identified by TCCD and differentiated according to real anatomical conditions. In addition, a separate analysis of flow responses to visual stimulation in selected cortical branches of the PCA has not been published previously.

In our study, the segments P1 to P3 were visualized in nearly all of our cases. Similar high detection rates of $72-100 \%$ have been reported for P1 and P2 [12-15]. Data for the cortical branches, however, were missing. The 2 proximal branches, the ATA and OTA, can be reliably identified with non-contrast-enhanced TCCD. The detection rates of $82 \%$ (ATA) and 96\% (OTA) correspond with the anatomical data of Zeal who reported rates of $84 \%$ and $96 \%$, respectively [5]. In contrast, the detection rates differ markedly in regard to the POA and CA with detection rates in our study of $69 \%$ and $62 \%$ but an anatomical presence in $96 \%$ and $100 \%$, respectively [5]. The main reason for this may be the restriction of insonation quality through the temporal bone for the visualization of more dorsally located vessels.

Regarding flow rate response to visual stimulation, our reported BFVs in P1 and P2 correspond to the data from the literature [12-14]. The highest cortical branch BFVs with closed eyes were found in the POA, followed by the OTA, CA and ATA. The vessel diameter is correlated to the significance of the artery and absolute BFV values correspond to the amount of perfused brain parenchyma by the selected artery. The perfusion area of the POA includes large parietal and occipital areas and that of the OTA temporal and occipital areas whereas the ATA is more restricted to anterior parts of the temporal lobe and the hippocampus. The wide inter-individual range in BFV in selected branches indicates the high variability in the perfusion area. This has been shown in anatomical studies for the OTA and CA [16]. Our findings are in good accordance with the anatomical literature. Diameter measurements of the POA, OTA, CA and ATA were reported to be $1.6 \mathrm{~mm}, 1.6 \mathrm{~mm}, 1.4 \mathrm{~mm}$ and $1.3 \mathrm{~mm}$, respectively [5]. Similar data of $1.5 \mathrm{~mm}, 1.6 \mathrm{~mm}, 1.3 \mathrm{~mm}$ and $1.2 \mathrm{~mm}$ were reported recently.

Like functional magnetic resonance imaging (fMRI) and positron emission tomography (PET), ultrasound allows the assessment of changes in local neuronal activity by measuring changes in blood flow velocities in a selected vessel segment, which corresponds to changes in regional blood flow (rCBF). In contrast to MRI and PET, ultrasound methods have a high temporal resolution allowing instant assessment of regional CBF changes. The major disadvantage of functional ultrasound, however, is the limited spatial resolution. Despite this, a large number of studies using TCD focusing on sensory, motor and cognitive functions, especially concerning language lateralization in man have been published in recent years $[17,18]$. An evoked flow response of PCA main stem flow to visual stimuli has been demonstrated using TCD shortly after its introduction in clinical medicine with a mean BFV increase of about $20 \%$ in healthy subjects [19-22]. PCA visual evoked flow has been used to separate PCA signals from vessels of the anterior circulation and from the superior cerebellar artery (SCA) [23] and to distinguish patients in a vegetative state [24].

However, data on flow responses in select cortical branches has not previously been reported. Our results show that the major evoked increase in diastolic BFVs after visual stimulation was as expected in the CA with $41.6 \%$ followed by the POA and OTA with $27.4 \%$ and $16.2 \%$, respectively. The weakest effect was observed in the ATA with an increase of $8.5 \%$ only. The obvious response in the POA and less in the OTA may be explained by their contribution to the perfusion of the striatal and peristriatal cortex in $20-35 \%$ and $3-22.5 \%$ of cases, respectively. The P2 segment showed an increase of diastolic BVF of $29.5 \%$, which corresponds to discussed data from the literature. [25-26]

Our observations may be doubly useful. First, the new TCCD vessel nomenclature allows a more exact anatomical description of PCA vessel anatomy and pathology. Instead of mere detection of main stem PCA pathology $[27,28]$, the newly described PCA segments may facilitate the recognition of vessel stenoses in more distal parts and in selected branches. It may also facilitate a more precise definition of main stem occlusions and a more elaborate description of collateral leptomeningeal PCA flow in MCA occlusion [25,26].

Second, the ability to examine select cortical branches may open new possibilities in functional Doppler of the visual system. One major obstacle of TCCD is that it allows only hand-held examination of the vessels, limiting the measurement of BFV changes during the visual task. Here, either newly developed TCCD probe holders, or information transfer (depth, probe position, wave form, $\mathrm{BFV}$, and flow direction) from TCCD to TCD, might be a solution.

Limitations of our study have to be mentioned. One is the subjects' relatively young age. A lower frequency of successful visualization in older subjects is expected due to a diminished temporal acoustic window. The use of echo-contrast agents can ameliorate the sensitivity of TCCD markedly [29]. However, the use of a contrast agent may require that different reference values are used [30]. Also, variations of PCA cortical branches have to be considered, e.g., a common temporal branch which bifurcates or trifurcates into several smaller branches, a middle temporal artery between the ATA and OTA, or a small hippocampal artery present as the first cortical branch of the PCA [5], leading to confusion with the ATA. Also a prominent posterior choroid or posterior pericallosal artery may arise from the P2 and P3 segments [23]. Finally, the main stem of the PCA may be confused with the SCA, which runs parallel and close together with the PCA at their origins from the basilar artery [23].

\section{Conclusion \\ $\nabla$}

Non-contrast-enhanced TCCD insonation through the temporal bone window allows visualization not only of the PCA main stem from the P1 to the P3 segments but also the cortical PCA branches, with the proximal ATA and OTA more readily visualized than the peripherally located POA and CA. The above nomenclature allows more accurate assessment of vascular disease location. Evoked flow velocity responses to a visual stimulus can be analyzed in PCA branches. Different reaction patterns can be used to distinguish branches, e.g., the POA from the CA, and open the door for new functional testing in cognitive and visual disorders.

\section{Affiliations}

${ }^{1}$ Department of Neurology, Institute for Clinical Sciences, Malmö, Sweden ${ }^{2}$ Neurology, Charité Berlin, Berlin, Germany

${ }^{3}$ Department of Neurology and Neurophysiology, AMEOS Klinikum Neustadt, Neustadt i. H., Germany

${ }^{4}$ Department of Neurology, Charité Campus Virchow, Berlin, Germany

${ }^{5}$ Neurologie, Neurologisches Zentrum der Segeberger Kliniken, Bad

Segeberg, Germany 


\section{References}

1 Brandt T, Steinke $W$, Thie A et al. Posterior cerebral artery territory infarcts: clinical features, infarct topography, causes and outcome. Multicenter results and a review of the literature. Cerebrovasc Dis 2000; 10: 170-182

2 Lee E, Kang DW, Kwon SU et al. Posterior cerebral artery infarction: diffusion-weighted MRI analysis of 205 patients. Cerebrovasc Dis 2009; 28: 298-305

3 Yasargil M. Microneurosurgery, Vol I: Microsurgical anatomy of the basal cisterns and vessels of the brain, diagnostic studies, general operative techniques and pathological considerations of the intracranial aneurysms. Thieme; 1984

4 Hoksbergen AW, Legemate DA, Ubbink DT et al. Collateral variations in circle of willis in atherosclerotic population assessed by means of transcranial color-coded duplex ultrasonography. Stroke 2000; 31: $1656-1660$

5 Zeal AA, Rhoton AL Jr. Microsurgical anatomy of the posterior cerebral artery. J Neurosurg 1978; 48: 534-559

6 Caruso G, Vincentelli F, Rabehanta P et al. Anomalies of the P1 segment of the posterior cerebral artery: early bifurcation or duplication, fenestration, common trunk with the superior cerebellar artery. Acta Neurochir (Wien) 1991; 109: 66-71

7 Lang J. A rare variation of the origin of the posterior cerebral artery (a. occipitalis lateralis from the internal carotid artery). J Hirnforsch 1982; 23: 301-310

8 Villablanca JP, Nael K, Habibi R et al. 3T contrast-enhanced magnetic resonance angiography for evaluation of the intracranial arteries: comparison with time-of-flight magnetic resonance angiography and multislice computed tomography angiography. Invest Radiol 2006; 41: 799-805

9 Forsting $M$. CTA of the ICA bifurcation and intracranial vessels. Eur Radiol 2005; 15 (Suppl 4): D25-D27

10 Tegeler CHBV, Gomez CR. Neurosonology. Mosby; 1996

11 Valdueza JM, Schreiber SJ, Roehl J-E et al. Neurosonology and Neuroimaging of Stroke. Georg Thieme Verlag; 2008

12 Baumgartner RW, Schmid C, Baumgartner I. Comparative study of power-based versus mean frequency-based transcranial color-coded duplex sonography in normal adults. Stroke 1996; 27: 101-104

13 Hoksbergen AW, Legemate DA, Ubbink DT et al. Success rate of transcranial color-coded duplex ultrasonography in visualizing the basal cerebral arteries in vascular patients over 60 years of age. Stroke 1999; 30: $1450-1455$

14 Martin PJ, Evans DH, Naylor AR. Transcranial color-coded sonography of the basal cerebral circulation. Reference data from 115 volunteers. Stroke 1994; 25: 390-396

15 Postert T, Meves S, Bornke C et al. Power Doppler compared to colorcoded duplex sonography in the assessment of the basal cerebral circulation. J Neuroimaging 1997; 7: 221-226
16 Marinkovic SV, Milisavljevic MM, Lolic-Draganic V et al. Distribution of the occipital branches of the posterior cerebral artery. Correlation with occipital lobe infarcts. Stroke 1987; 18: 728-732

17 Deppe M, Knecht S, Papke K et al. Assessment of hemispheric language lateralization: a comparison between fMRI and fTCD. J Cereb Blood Flow Metab 2000; 20: 263-268

18 Duschek S, Schandry R. Functional transcranial Doppler sonography as a tool in psychophysiological research. Psychophysiology 2003; 40: 436-454

19 Sturzenegger $M$, Newell DW, Aaslid R. Visually evoked blood flow response assessed by simultaneous two-channel transcranial Doppler using flow velocity averaging. Stroke 1996; 27: 2256-2261

20 Aaslid $R$. Visually evoked dynamic blood flow response of the human cerebral circulation. Stroke 1987; 18: 771-775

21 Diehl B, Knecht S, Deppe $M$ et al. Cerebral hemodynamic response to generalized spike-wave discharges. Epilepsia 1998; 39: 1284-1289

22 Gomez SM, Gomez CR, Hall IS. Transcranial Doppler ultrasonographic assessment of intermittent light stimulation at different frequencies. Stroke 1990; 21: 1746-1748

23 Pade 0, Schreiber SJ, Eggers J et al. Superior cerebellar artery - a potential pitfall in transcranial posterior cerebral artery insonation. Ultraschall Med 2010; 31: 401-404

24 Becker VU, Hansen HC, Brewitt U et al. Visually evoked cerebral blood flow velocity changes in different states of brain dysfunction. Stroke 1996; 27: 446-449

25 Gomez-Choco M, Valdueza JM. Letter by Gomez-Choco and Valdueza regarding article, "posterior cerebral artery laterality on magnetic resonance angiography predicts functional outcome". Stroke 2013; 44: e51

26 Kim Y, Sin DS, Park HY et al. Relationship between flow diversion on transcranial Doppler sonography and leptomeningeal collateral circulation in patients with middle cerebral artery occlusive disorder. J Neuroimaging 2009; 19: 23-26

27 Baumgartner RW, Mattle HP, Schroth G. Assessment of $>/=50 \%$ and $<50 \%$ intracranial stenoses by transcranial color-coded duplex sonography. Stroke 1999; 30: 87-92

28 Kimura K, Minematsu K, Yasaka $M$ et al. Evaluation of posterior cerebral artery flow velocity by transcranial color-coded real-time sonography. Ultrasound Med Biol 2000; 26: 195-199

29 Gerriets T, Goertler M, Stolz E et al. Feasibility and validity of transcranial duplex sonography in patients with acute stroke. J Neurol Neurosurg Psychiatry 2002; 73: 17-20

30 Yokoyama $N$, Schwarz KQ Chen $X$ et al. The effect of echo contrast agent on Doppler velocity measurements. Ultrasound Med Biol 2003; 29: 765-770 